\title{
TTR
}

Traduction, terminologie, re?daction

\section{Langues étrangères et savoir romantique : considérations préliminaires}

\section{Rainier Grutman}

Volume 9, numéro 1, 1er semestre 1996

Le festin de Babel

Babel's Feast

URI : https://id.erudit.org/iderudit/037239ar

DOI : https://doi.org/10.7202/037239ar

Aller au sommaire du numéro

\section{Éditeur(s)}

Association canadienne de traductologie

ISSN

0835-8443 (imprimé)

1708-2188 (numérique)

Découvrir la revue

Citer cet article

Grutman, R. (1996). Langues étrangères et savoir romantique : considérations préliminaires. TTR, 9(1), 71-90. https://doi.org/10.7202/037239ar
Résumé de l'article

Langues étrangères et savoir romantique : considérations préliminaires - La valeur et la signification de la cohabitation textuelle des langues changent trop au gré des attitudes épilinguistiques pour qu'il soit possible, ou même salutaire, de les subsumer sous le dénominateur commun du " réalisme ». La lecture référentielle, en effet, ne rend guère compte des manières dont les langues se font écho, se complètent, se traduisent à l'intérieur de l'oeuvre littéraire. Or, loin de simplement enregistrer le social, celle-ci formule un savoir sur les idiomes mis à contribution, ce qu'illustre une brève comparaison des conceptions " classique » et " romantique » du plurilinguisme (à partir du Pantagruel de François Rabelais et Notre-Dame de Paris de Victor Hugo). Alors que dans le premier cas, le xénisme fait obstacle en court-circuitant le rapport dénotatif entre les mots et les choses, dans le deuxième, il s'insère dans un réseau de connotations propres au texte.
Tous droits réservés (C) TTR: traduction, terminologie, rédaction — Les auteurs, 1996
Ce document est protégé par la loi sur le droit d'auteur. L'utilisation des services d'Érudit (y compris la reproduction) est assujettie à sa politique d'utilisation que vous pouvez consulter en ligne.

https://apropos.erudit.org/fr/usagers/politique-dutilisation/ 


\section{Langues étrangères et savoir romantique : considérations préliminaires}

\section{Rainier Grutman}

$\mathrm{Au}$ départ de ma réflexion, se trouve l'idée que la cohabitation textuelle des langues, thème du présent numéro, n'apparaît pas n'importe quand ni n'importe où, mais acquiert une valeur et une signification particulières selon les attitudes, épilinguistiques notamment, qui prévalent dans un état de société. L'idée serait triviale si elle ne venait contredire nos habitudes de lecture (et de traduction), lesquelles font l'impasse sur les rapports ambigus que le plurilinguisme des textes peut entretenir avec la polyglossie externe, référentielle. Aussi, avant de pouvoir envisager le " savoir " des langues mis en discours à l'époque romantique, il convient d'expliquer ce que j'entends par textualisation des langues ou hétérolinguisme et de revenir brièvement sur l'esthétique réaliste. Ce détour s'impose d'autant plus que le débat s'est quelque peu enlisé dans une discussion sur la vraisemblance et ses limites, elle-même promue par les cuvres canoniques du $\mathrm{XIX}^{\mathrm{e}}$ siècle.

\section{Illusion référentielle et savoir des textes}

Tout texte utilise forcément un nombre limité de langues, qu'à vrai dire, il agence plus qu'il ne les juxtapose. Rendre compte de ces stratégies textuelles en termes d'《 hétérolinguisme ", plutôt que de 
" bilinguisme " ou de " diglossie ${ }^{\mathrm{l}}$ ", présente plusieurs avantages. D'abord, grâce à son hybridité étymologique, le néologisme composé du grec heteros (" autre ») et du latin lingua (" langue ») met en abyme le mélange et le métissage qu'il désigne. Ensuite et surtout, le concept concerne spécifiquement les textes, contrairement aux termes psycho- ou sociolinguistiques, qui s'appliquent aux auteurs et aux lecteurs, non aux cuvres, avec lesquelles ils entretiennent un rapport indirect : ainsi parle-t-on de l'influence du contexte sur le style d'un écrivain ou de la structure linguistique propre à l'univers fictif du texte. Par hétérolinguisme, j'entends plutôt la textualisation d'idiomes étrangers aussi bien que de variétés (sociales, régionales, chronologiques) de la langue auctoriale. Le bilinguisme textuel et la diglossie textuelle en seraient des modalités spécifiques.

Compris dans ce sens, l'hétérolinguisme ne respecte pas forcément les données extra-textuelles. Sans le juger exclusivement à l'aune de la polyglossie sociale, accordons-lui cette liberté, cette marge de mancuvre. Ainsi, pour ne donner qu'un exemple bien connu, au lieu de toujours rapprocher le baron Frédéric de Nucingen de tel banquier qui défrayait la chronique sous la monarchie de Juillet, on ferait observer que l'accent du personnage balzacien a été systématisé aux fins de la caricature, et l'on en tirerait les conséquences qui s'imposent. Car si la tendance à fermer les voyelles et à neutraliser l'opposition phonologique entre consonnes sourdes et consonnes sonores caractérise effectivement l'accent

1. Au sujet de la " diglossie ", on pourra consulter, entre autres, les utiles mises au point de Georg Kremnitz (1987) et de Lambert-Félix Prudent (1981). Ailleurs (Grutman 1990, 1993), je me suis servi du terme "bilinguisme " en lui donnant un sens technique. Devant le tollé que soulève son emploi au Québec, je l'ai remplacé par " hétérolinguisme ", lequel mot sonne, hélas! pédant à cause des voies étymologiques qu'emprunte la néologie française. 
tudesque, elle est rarement aussi absolue que chez Nucingen ${ }^{2}$. Le portrait linguistique de ce dernier ne dénote aucune réalité, mais combine des éléments doxiques de la germanophobie avec la psychologie sommaire du théâtre burlesque. Croire qu'un James de Rothschild aurait prononcé des phrases comme « Puisqui matame fous encache, [...] fous êtes sir d'être pien ressi " (Balzac, 1963, p. 146), c'est négliger le dialogue intertextuel qui travaille tout texte et rester insensible aux qualités connotatives du langage littéraire naguère mises en évidence par Roland Barthes (1970, pp. 13-16 et 1982 , p. 89).

Que la version des textes s'écarte ainsi de celle des linguistes ne doit pas surprendre dans la mesure où, y compris dans le roman réaliste du siècle dernier, les masses référentielles sont agencées afin de faire ressortir l'un ou l'autre aspect du monde représenté. En littérature, la réduction quantitative du contenu est souvent inversement proportionnelle à l'exploitation qualitative de l'expression. Dans son introduction à Pierre et Jean, en 1887, Guy de Maupassant ne disait pas autre chose quand il proposait d'appeler "Illusionnistes » les écrivains qui réussissent dans l'art réaliste :

Raconter tout serait impossible, car il faudrait alors un volume au moins par journée, pour énumérer les multitudes d'incidents insignifiants qui emplissent notre existence. [...] Voila pourquoi l'artiste, ayant choisi son thème, ne prendra dans cette vie encombrée de hasards et de futilités que les détails

2. C'est ce qu'avait déjà noté Hans Băckvall (1970, p. 384), dans un article qui a bien des mérites d'un point de vue heuristique, mais n'en pèche pas moins par positivisme. Sa propre mise en garde - " il ne faut pas toujours prendre au sérieux" le pseudo-allemand chez Balzac, " reproduit au moyen d'une orthographe souvent assez gauche et fantasque " et dont le phonétisme paraît " fort exagéré " (p. 384) - ne l'empêche point de proposer cinq " prototypes éventuels de Nucingen 》 (p. 392), à savoir, outre les financiers Rothschild et Humann, l'anatomiste Gall, l'ancien forçat Vidocq et... madame Hanska. 
caractéristiques utiles à son sujet, et il rejettera tout le reste, tout l'à-côté. (1971, pp. 370-371)

Il en va de même de l'hétérogénéité langagière d'œuvres dont l'histoire traverse plusieurs couches sociales, plusieurs pays, voire plusieurs époques. Les langages propres à chaque situation ne pouvant être tous compris du public, une sélection s'impose, de sorte que la polyglossie du monde externe sera seulement évoquée à l'aide de quelques touches soigneusement apportées : « Das Fremdwort verfremdet, wenn es sparsam gebraucht wird ", rappelle à bon escient András Horn (1981, p. 240). En plus d'imiter des phénomènes linguistiques observables dans la réalité, les xénismes peuvent encore caractériser (ridiculiser ?) un personnage ou les membres d'un groupe, introduire un niveau métalinguistique ou camoufler des sous-entendus scatologiques ${ }^{3}$. Aussi m'est-il difficile de souscrire sans réserves à une vision de la littérature qui partirait du dogme suivant : "The interlingual tension between language as represented object (within the original or reported speech-event) and language as representational means (within the reporting speechevent) is primarily mimetic" (Meir Sternberg, 1981, p. 222, voir également Kathleen N. March, 1984). Il ne s'agit certes pas de nier que la lisibilité est largement assurée par la corrélation linguistique d'un texte avec la vie sociale, ni que ce déchiffrement se fait sur un mode peu ou prou dénotatif; mais la croyance à un rapport réciproque, bijectif, entre l'hétérolinguisme montré dans les textes et le plurilinguisme extra-textuel a parfois fait naïvement confondre la carte avec le terrain. Bien qu'en vérité, il fasse écran, le langage littéraire continue de faire illusion.

Si les pages précédentes font comprendre l'intérêt qu'il y aurait à sortir l'étude du plurilinguisme textuel des ornières du réalisme et de la vraisemblance, l'on évitera cependant de jeter le

3. Flaubert le savait fort bien, qui notait dans son Dictionnaire des idées reçues (s.v. " latin $n)$ : " Il faut se méfier des citations en latin; elles cachent toujours quelque chose de leste " $(1979$, p. 536). 
bébé avec l'eau du bain en écartant tout simplement la mimésis. Mieux vaut lui adjoindre un point de vue connotatif, plus apte à rendre compte des manières dont les langues se font écho à l'intérieur du texte et soulignent l'auto-référentialité de ce dernier. En effet, loin de se contenter d'enregistrer la rumeur sociale, les œuvres littéraires formulent un savoir sur les idiomes mis à contribution. La « clef » de l'hétérolinguisme serait par conséquent moins à chercher du côté de l'auteur et de sa compétence linguistique - comme le veut un malentendu fort répandu - que đans le degré d'intégration textuelle des éléments allophones. C'est ici que surgit la problématique de la traduction (au sens large), celle-ci étant une stratégie d'écriture utilisée au moins depuis l'Antiquité afin d'amortir le choc sonore créé par les verba peregrina à l'intérieur des textes. Or, sì, à première vue, les traductions sont là pour ménager le lecteur monolingue, il faut bien voir que leur présence/absence permet d'obtenir toute une gamme d'effets. Un exemple illustrera mon propos. Dans Women in Love (1921) de D.H. Lawrence, Ursula Brangwen appelle l'attitude dominatrice du matou Mino « a lust for bullying - a real Wille zur Macht - so base, so petty ", ce qui amène Rupert Birkin à faire l'observation suivante:

I agree that the Wille zur Macht is a base and petty thing. But with the Mino, it is the desire to bring this female cat into a pure stable equilibrium, a transcendent and abiding rapport with the single male. Whereas without him, as you see, she is a mere stray, a fluffy sporadic bit of chaos. It is a volonté de pouvoir, if you like, a will to ability, taking pouvoir as a verb. (D.H. Lawrence, 1960, p. 167)

Les traductions anglaises ainsi obtenues, l'une directement de l'allemand, l'autre de manière indirecte, à partir du français, ne sont guère synonymes, en dépit de la " source " nietzschéenne qu'elles ont en commun. Il y a là, de la part de Lawrence, une leçon que l'on ne saurait prendre à la légère, à savoir que la dimension connotative (même si elle s'avère stéréotypée) est inhérente au langage. Tandis que les sons rugueux de l'allemand suggèrent une certaine violence, la langue française tend au verbiage rhétorique, 
comme Ursula ne manque pas de le souligner dans sa réponse laconique: "Sophistries! 》 (p. 167).

On pourrait multiplier les exemples sans pour autant réussir à en dégager une poétique universelle. La matière est trop abondante, et l'on serait tenté de faire comme Wilhelm Theodor Elwert (l'un des premiers chercheurs à étudier la question de façon plus ou moins systématique), qui conclut, après avoir donné du plurilinguisme linguistique " un répertoire imposant, mais dont il serait facile de signaler les lacunes » : " autant d'auteurs, autant de problèmes différents » $(1960$, p. 415$)$. Il semble au moins possible de décrire les modalités de l'hétérolinguisme pour un corpus circonscrit dans le temps et dans l'espace, caractérisé par un horizon épistémique et des conditions sociales relativementhomogènes. C'est dans cet esprit qu'il faut comprendre le parallèle qui suit ${ }^{4}$.

\section{Paradigme classique et mutation romantique}

À l'époque classique - laquelle s'achève grosso modo entre 1775 et 1825 selon Michel Foucault (1966, p. 233) - , la dénotation règne en maitresse absolue, les mots quadrillent la connaissance des choses. Loin d'être, comme au Moyen-Âge, " lié à ce qu'il marque par les liens solidaires et secrets de la ressemblance " (Foucault, 1966, p. 72), le signe linguistique représente dans un espace combinatoire, dans un dédoublement logique. Telle est déjà, au demeurant, l'opinion de François Rabelais, en qui l'on ne soupçonnerait guère un précurseur du classicisme pourtant. Dans le dix-neuvième chapitre du Tiers Livre, Pantagruel refuse de qualifier nos langues de naturelles, parce qu'elles « sont par institutions

4. Dans des travaux ultérieurs, je me propose d'examiner la théorie et la pratique romantiques du plurilinguisme en France. Ce projet, dont l'exposé que voici constitue l'amorce, bénéficie de l'appui du Conseil de recherches en sciences humaines du Canada et de l'École des études supérieures et de la recherche de l'Université d'Ottawa, organismes qui sont ici remerciés. 
arbitraires et convenences des peuples ${ }^{5}$ ». Du point de vue du plurilinguisme, pareille conception du rapport entre les mots et les choses présente un inconvénient majeur. Sans une maîtrise adéquate du sociolecte ou de la langue étrangère, il est impossible de déchiffrer ce qui ressemblera forcément à un idiome inintelligible. Aussi tente-t-on de préserver l'ordre du discours dans la littérature de l'âge classique, tantôt en réduisant l'univers référentiel (c'est le cas de la société de cour dans la Princesse de Clèves de Madame de Lafayette), tantôt en peuplant les textes de polyglottes surdoués (on songe à l'Ingénu de Voltaire, par exemple).

Chez Rabelais, par contre, la mise en scène de la confusion produite par l'impossibilité de communiquer en dehors des conventions entérinées par la société témoigne d'une étonnante lucidité. Dans le neuvième chapitre de son Pantagruel, le géant éponyme aperçoit un homme « pitoyablement navré en divers lieux, et tant mal en ordre qu'il semblait être échappé es chiens " (Rabelais, 1994, p. 351). Quand il s'enquiert de l'identité, de la provenance et de la destinée du quidam, celui-ci lui répond en non moins de dix langues étrangères et trois idiomes factices, soit, dans cet ordre : l'allemand, l'antipodien, le toscan, l'écossais, le basque, le lanternois, le néerlandais, le castillan, le danois, l'hébreu, le grec, l'utopien et le latin. Or les passages polyglottes resteront lettre morte pour l'entourage de Pantagruel. Cela paraît d'autant plus étonnant que la personne entière de l'inconnu exprime sa détresse et que, comme le souligne ce dernier dans son discours grec, les paroles ne s'imposent que là où les faits ne se montrent pas clairement (Rabelais, 1994, p. 357). Il faut attendre la fin du chapitre pour apprendre que le " gentil compaignon " se nomme Panurge; que,

5. Rabelais (1994), p. 663. Répété jusqu'au milieu du siècle des Lumières (par Locke en Angleterre, par Condillac en France), le constat devait réapparaître au début du nôtre, sous la plume de Ferdinand de Saussure, après une éclipse assez longue pour faire croire à une innovation radicale de la part du linguiste genevois. Hans Aarsleff (1982, pp. 363-364) signale les Idéologues et Hippolyte Taine comme intermédiaires possibles. 
Tourangeau, il a le français comme langue maternelle, et qu'enfin, il désire se désaltérer et se nourrir : " dents agûes, ventre vide, gorge sèche, tout y est délibéré; si me voulez mettre en œuvre, ce sera basme de me voir briber » $(1994$, p. 359$)$.

En regardant de plus près ce dialogue de sourds, on s'aperçoit que l'opacité n'y est pas le lot de tous les idiomes, bien au contraire. Nul ne s'offusquera de ce que le « langaige maternel françois ", promu au rang de langue d'État par l'Ordonnance royale de Villers-Cotterêts en 1539 (soit sept ans après la parution de Pantagruel), serve déjà de point de ralliement, de lingua franca. Mais que dire du fait que ni l'italien - la langue par excellence de la Renaissance - ni l'espagnol - alors propagé avec un zèle extraordinaire par les troupes de Charles Quint - ne soient à la portée du public de Panurge ? Et que déduire de la re-connaissance (à défaut d'une connaissance véritable) de parlers aussi exotiques que le basque, le Scots, le danois et, chose étonnante, de toutes les langues imaginaires? Quelle que soit leur importance scientifique, culturelle ou politique, la plupart des idiomes convoqués par Panurge laissent penauds ses interlocuteurs : " dea, mon ami ", lui enjoint Pantagruel, " je ne fais aucun doute que ne sachez bien parler divers langages; mais dites-nous ce que voudrez en quelque langue que puissions entendre " (1994, p. 355). Aucun des codes dont Gargantua venait de recommander l'étude dans le chapitre précédant immédiatement celui qui retient mon attention - le latin scolastique, le grec humaniste, l'hébreu biblique - n'est un moyen de communication plus efficace que, par exemple, le langage des Antipodes. Leur inutilité sera confirmée par la réponse française : en plus de donner la solution de l'énigme, Panurge y reprend les mêmes thèmes (la faim, la soif, la misère), la commence également par une apostrophe déférente à l'égard de son interlocuteur (terminant ainsi la série Juncker, Signor mio, Lard, Jona, Heere, Seignor, Myn herr, Adoni, Despota) et invoque l'autorité divine. Se répétant entre elles, ajoutant peu au texte français, faisant de surcroît double emploi avec l'aspect physique de Panurge, les élucubrations hétérolingues s'avèrent triplement redondantes. 
Étonnante leçon pour l'historien de la linguistique! Rabelais émet ante litteram des réserves quant à la prétendue primauté de la pensée universelle sur la forme qu'elle revêt dans les langues individuelles. Prenant le contre-pied de ce qui sera, un siècle plus tard, la position des jansénistes de Port-Royal dans leur Grammaire générale et raisonnée (1660), le médecin chinonais nous présente une " pathologie de la communication " (Bastiaensen, 1974, p. 552) qui néglige la fonction référentielle du langage au profit, notamment, de sa fonction phatique. En effet, puisque les compagnons de Pantagruel n'entendent de toute manière que le seul français, pourquoi Panurge s'ingénie-t-il à leur demander l'aumône en tant de langues, et donc, de rester (littéralement) sur sa faim, sinon pour prolonger le contact? Mais il y a plus. En présentant la même information sous une forme chaque fois neuve, le glossolale attire l'attention de Pantagruel sur le signifiant, pour mieux ériger celui-ci en barrière, en obstacle à la compréhension, annonçant ainsi sa fétichisation future de la part des romantiques.

Cependant, pour pouvoir parler d'une doctrine romantique des langues, il ne suffit pas que soient associés son et sens; il faut encore que le son fasse sens, c'est-à-dire que le signifiant devienne transparent et révèle le signifié. Le romantisme se caractérise, entre autres choses, par une volonté de sonder le tréfonds de l'âme humaine, par une recherche inlassable de la vérité première, ensevelie par le passage du temps. Chez de nombreux romantiques, hommes de science aussi bien qu'hommes de lettres, on trouve un même fantasme du déchiffrement et de la mise au jour. Voulant tout expliquer, ils pratiquent volontiers une dérive exégétique qui n'évite

6. On aura noté que Panurge critique le conventionnalisme défendu plus haut par Pantagruel. Loin d'être irréductible, l'opposition entre les deux personnages structure une bonne partie de l'œuvre rabelaisienne. Elle a été étudiée du point de vue langagier par François Rigolot (1976), mais ne se limite pas à ce domaine (Michel Jeanneret, 1984). Les pages qui préceddent condensent une analyse faite dans Grutman (1993, pp. 218-223). 
pas toujours la Hineininterpretierung, le délire de l'interprétation. Dans une telle optique, tout signe - et, a plus forte raison, tout signe d'un idiome étranger - peut devenir un hiéroglyphe, un caractère sacré, un mot à valeur divine et divinatoire. En 1794, l'auteur fort sérieux d'une Doctrine des nombres de la nature [...], une clef pour les hiéroglyphes formule l'idée comme suit : " Chaque objet possède sa propre bouche pour la révélation de son dedans et de son dehors; c'est en cela que consiste la langue de la nature, la connaissance des signatures des choses. " (Eckartshausen, cité par Gusdorf, 1993, p. 852) Mais on la rencontre également dans les célèbres premières pages d'une Étude philosophique publiée par Balzac en 1832 et où il prête à son personnage Louis Lambert le discours que voici:

Quel beau livre ne composerait-on pas en racontant la vie et les aventures d'un mot ? [...] À le regarder, abstraction faite de ses fonctions, de ses effets et de ses actes, n'y a-t-il pas de quoi tomber dans un océan de réflexions? La plupart des mots ne sont-ils pas teints de l'idée qu'ils représentent extérieurement? [...] L'assemblage des lettres, leurs formes, la figure qu'elles donnent à un mot, dessinent exactement, suivant le caractère de chaque peuple, des êtres inconnus dont le souvenir est en nous. Qui nous expliquera philosophiquement la transition de la sensation à la pensée, de la pensée au verbe, du verbe à son expression hiéroglyphique, des hiéroglyphes à l'alphabet, de l'alphabet à l'éloquence écrite [...] ? L'antique peinture des idées humaines configurées par des formes zoologiques n'aurait-elle pas déterminé les premiers signes dont s'est servi l'Orient pour écrire ses langages ? [...] Est-ce à cet ancien Esprit que nous devons les mystères enfouis dans toute parole humaine? (Balzac, 1927, pp. 48-49)

La référence aux hiéroglyphes traduit l'engouement pour l'hermétisme auquel on assiste en Europe depuis la découverte, par William Jones, du sanskrit (l'ancienne langue sacrée des Indiens que Frédéric von Schlegel considérait comme la langue-matrice de l'humanité entière). $\grave{A}$ peine une génération plus tard, Thomas Young et le jeune Champollion percent le secret de la pierre de Rosette. Ces tours de force répétés, on s'en doute, frappent l'imagination des écrivains. Bientôt, ils partagent la fascination des 
philologues pour le " mot étranger cryptique " (Bakhtine et Volochinov, 1977, p. 108). Victor Hugo ne fit guère exception, comme le montrera un bref examen de deux textes contemporains de celui de Balzac : le Dernier jour d'un condamné et Notre-Dame de Paris.

\section{Hugo hétérolingue}

La veille de son exécution, le condamné imaginé par Hugo promène sa lampe sur les murs de sa cellule, qui sont autant de pages " couvert[e]s d'écritures, de dessins, de figures bizarres, de noms qui se mêlent et s'effacent les uns les autres " (1985, p. 441). Rapidement, la trace des absents acquiert sa propre épaisseur matérielle: " C'est du crayon, de la craie, du charbon, des lettres noires, blanches, grises [...], çà et là des caractères rouillés qu'on dirait écrits avec du sang. " (1985, p. 441) Par métonymie, les signes renvoient à leur origine, à l'instrument qui les a tracés, voire à l'absence de tout instrument (le sang des doigts). Par métaphore, ils se substituent aux corps décapités de leurs auteurs. C'est dire que la rhétorique ordonne ce qui paraît désordonné : les graffiti peuvent non seulement être reconstitués (selon les règles de la philologie), mais encore interprétés (conformément aux procédures de l'herméneutique).

Certes, si j'avais l'esprit plus libre, je prendrais intérêt à ce livre étrange qui se développe page à page à mes yeux sur chaque pierre de ce cachot. J'aimerais à recomposer un tout de ces fragments de pensée, épars sur la dalle; à retrouver chaque homme sous chaque nom; à rendre le sens et la vie à ces inscriptions mutilées, à ces phrases démembrées, à ces mots tronqués, corps sans tête comme ceux qui les ont écrits. (Hugo, 1985 , p. 441)

Dans ce passage où le savoir unifie et ramène le sens qui se dérobe, le narrateur se sert de ce que Mikhaîl Bakhtine et Valentin Volochinov ont appelé le " sifflet de la philologie ", dont c'est précisément la fonction "d'éveiller les morts " (1977, p. 104), d'insuffler une nouvelle vie aux langues figées dans la pierre. 
Quand paraît le Dernier jour d'un condamné, en février 1829 , Hugo nourrit déjà un autre projet de roman. La philologie et l'hétérolinguisme y tiendront une place à la fois plus grande et plus ambigué. Notre-Dame de Paris, la chose est connue, s'ouvre sur la découverte d'un hiéroglyphe gravé à même le mur de la cathédrale, " dans un coin obscur de l'une des tours " (1985, p. 491). Or les lettres du mot grec anankè (fatalité) ont été calligraphiées en alphabet gothique, " comme pour révéler que c'était une main du moyen-âge qui les avait écrites" (p. 491). Contrairement à ce qui se passait chez Rabelais, où le xénisme faisait obstacle en courtcircuitant le rapport dénotatif entre les mots et les choses, ici, nous le verrons, il fournit la clef du livre en s'insérant dans un réseau de connotations.

Un chapitre situé au cœur du roman fait écho à l'inscription inaugurale. L'itinéraire de la lecture mène en effet, à travers le dédale de Notre-Dame, au cabinet secret où l'archidiacre Claude Frollo se livre à de coupables expériences alchimiques. Chercheur de la pierre philosophale, le clerc partage avec le philologue, outre la manie du décryptage, le raisonnement par analogie. En comparant les lettres communes à deux noms propres préalablement connus, sur la pierre de Rosette d'une part, sur l'obélisque de Philae d'autre part, Champollion fit jaillir le sens caché des hiéroglyphes phonétiques. De même, en rapprochant la lumière du soleil, le soleil du feu, et le feu de l'or, Frollo espère soutirer son secret à la nature (Hugo, 1985, p. 685). Le parallèle ne s'arrête pas là toutefois, puisque nous apercevons la cellule de dom Claude à travers les yeux de son frère Jehan, lequel voit sans être vu, n'ayant hasardé que sa tête par la porte entrebâillée. En l'occurrence, l'expédient thêâtral a pour effet de le/nous placer dans une position comparable à celle de l'égyptologue profanant la sépulture des pharaons, de sorte que ses yeux (et les nôtres) s'attardent spontanément sur les nombreuses légendes qui jonchent la chambre.

Du reste, lettres gothiques, lettres hébrafques, lettres grecques et lettres romaines, pêle-mêle; les inscriptions débordant au hasard, celles-ci sur celles-là, les plus fraîches effaçant les plus anciennes, et toutes s'enchevêtrant les unes dans les autres 
comme les branches d'une brousaille [...]. [L]e tout était traversé à tout propos par des étoiles, des figures d'hommes ou d'animaux et des triangles qui s'intersectaient, ce qui ne contribuait pas peu à faire ressembler la muraille barbouillee de la cellule à une feuille de papier sur laquelle un singe aurait promené une plume chargée d'encre. (Hugo, 1985, pp. 684685)

À la confiance du condamné de 1829 , reprenant à loisir « la lecture de [s]on mur " (Hugo, 1985, p. 442) et identifiant sans peine les noms qui s'y trouvent, succède la méfiance du narrateur de 1831 . Ce n'est pas que les signes soient derechef devenus conventionnels. Mais, par rapport au $\mathrm{XV}^{\boldsymbol{e}}$ siècle finissant (l'époque décrite dans Notre-Dame de Paris), les idiomes classiques ont gagné en opacité, étant désormais réservés aux seuls initiés, au prêtre et au philologue - qui souvent ne font qu'un d'ailleurs. Pour se frayer un chemin à travers la " brousaille " de symboles, il est en effet conseillé de pouvoir identifier et lire ceux-ci. Cela ne pose guère de difficultés dans le roman hugolien, lequel, système parfaitement autarcique, fournit lui-même la clef de l'énigme qu'il formule. Dès qu'on les considère en série, on s'aperçoit que les cryptogrammes de Frollo obéissent à une logique interne. Soit les exemples suivants: « SPIRA, SPERA [souffle, espère]; Unde ? inde ? [d'où ? de là ?]; Astra, castra [astres, camp], nomen, numen [nom, volonté divine] " (Hugo, 1985, pp. 684-685). Dans chaque cas, l'alchimiste a procédé par commutation phonologique, en regroupant des mots que distingue un seul phonème. Mais à cette homonymie partielle, il superpose une synonymie, dans la mesure où il réinterprète le sens des mots rapprochés à la lumière de leur morphologie. Autrement dit, son analyse repose sur la paronomase ${ }^{7}$, trope qu'affectionnait également

7. « La paronomase vient "motiver" la ressemblance des signifiants en suggérant une parenté des signifiés. Niant l'arbitraire du signe, [elle] suggère une affinité sémantique entre les mots phonétiquement proches. Le dicton "Qui se ressemble s'assemble" en énonce le principe et en constitue luimême une illustration. " (Klein-Lataud, 1991, p. 28) Parfois, 
le poète Hugo (en témoigne la pièce « Nomen, Numen, Lumen 》 dans les Contemplations). Libéré de la tutelle référentielle, le sens circule en circuit fermé, a l'intérieur du langage. De manière tout à fait moderne, " les mots deviennent texte à fracturer pour qu'on puisse voir émerger en pleine lumière cet autre sens qu'ils cachent " (Michel Foucault, 1966, p. 315).

D'un signifiant à l'autre, le romantique volontiers cratylien met la forme à contribution afin d'ouvrir l'éventail des possibilités sémantiques. En tant que lecteurs, nous sommes invités à lui emboîter le pas, et à restituer le sens des mots obscurs en nous inspirant de mots connus. Cela peut donner des résultats inattendus. Parmi les devises gravées sur le mur de Notre-Dame, il se trouve un vocable grec " dénué de tout sens apparent " (Hugo, 1985, p. 685), mais qui commence par les mêmes lettres qu'anankè. Les érudits allégueront avec raison que le mot en question, anagkophagia, figure sur la page de dictionnaire conservée dans les dossiers du roman Notre-Dame de Paris. Du point de vue de l'histoire littéraire, il constituerait une preuve du pédantisme qu'affectionnait le chef du Cénacle parisien. Ce faisant, on n'explique guère toutefois le fonctionnement textuel du xénisme, dont le manque de sens dénotatif produit en l'occurrence une sorte de débordement connotatif. La solution se trouve dans une série morphologique à laquelle appartiennent, outre les deux hellénismes déjà mentionnés, le mot biblique anagneia, signifiant "impureté » (p. 688), et arachnè, l'araignée, qui est chez Hugo le paronyme et le symbole d'anankè, de la fatalité. La première chose aperçue par Jehan dans la chambre de son frère est en effet « une ronde toile d'araignée, qui inscrivait avec goût sa rosace délicate dans l'ogive de la lucarne, et au centre de laquelle l'insecte architecte se tenait immobile comme le moyeu de cette roue de dentelle. " (p. 684) Au chapitre suivant, l'archidiacre, contemplant le spectacle d'une mouche qui se fait dévorer, s'exclame : « laissez faire la fatalité ", " laissez faire l'araignée » (p. 694).

Hugo recourt à une figure dérivée de la paronomase, tel le polyptote " homo homini monstrum » (1985, p. 685). 


\section{Pour conclure provisoirement}

Le xénisme romantique, on le constate, projette son ombre sur les mots qui l'entourent: un mot peut créer un monde. D'où son importance plus qualitative que quantitative dans l'esthétique romantique du plurilinguisme, laquelle s'arrête le plus souvent au niveau lexical. $\grave{A}$ l'instar des philologues qui furent ses contemporains, le poète romantique a horreur des signes opaques. Il veut les expliquer coute que coûte, quitte à les remotiver artificiellement, en recourant à l'étymologie (populaire) ou en provoquant des "collisions " paronymiques. Par voie de conséquence, l'attitude qui consiste à sauter les passages en langue étrangère, sous prétexte qu'on ne les comprend pas, n'est guère conforme au programme de lecture que proposent les romantiques. Elle nous condamnerait à laisser échapper une parcelle signifiante, et souvent significative, de leur cuvre.

Là semble d'ailleurs résider l'un des principaux paradoxes de l'étude du plurilinguisme en littérature. Si l'on considère celui-ci comme un phénomène avant tout formel, il est possible de le décrire à l'aide de principes stylistiques (Ulimann, 1964, pp. 44-64) ou dans le cadre d'une typologie (on trouvera des exemples dans Baetens Beardsmore, 1978; Goetsch, 1987 et Grutman, 1990). Tout en permettant d'étudier les corpus les plus variés et de prendre conscience de la très grande richesse du sujet, ces différentes grilles ne rendent pas forcément compte de sa complexité socio-historique. Comme elles ne sauraient prétendre à l'exhaustivité, les typologies demandent à être complétées par des lectures plus fines, plus respectueuses de l'idiosyncrasie des cuvres, d'autant plus que cellesci font appel à plusieurs procédés à la fois pour marquer

8. "Oui! poursuivit [Claude Frollo] en secouant la tête, voilà où en sont les études et les lettres maintenant. La langue latine est à peine entendue, la syriaque inconnue, la grecque tellement odieuse que ce n'est pas ignorance aux plus savants de sauter un mot grec sans le lire, et qu'on dit : Graecum est, non legitur " (Hugo, 1985, p. 688). 
l'intégration des idiomes étrangers. En définitive, si l'on se doute bien que ses effets ont évolué au fil des siècles - les analyses esquissées dans les pages précédentes donnent une idée des différences qui ont pu exister entre les conceptions classique et romantique -, l'histoire de l'hétérolinguisme comme stratégie textuelle reste à écrire.

\section{Références}

AARSLEFF, Hans (1982). From Locke to Saussure; Essays on the Study of Language and Intellectual History. London, Athlone Press.

BÄCKVALL, Hans (1970). " Le charabia 'tudesque' dans l'œuvre de Balzac. " Moderna spräk, pp. 383-395.

BAETENS BEARDSMORE, Hugo (1978), « Polyglot Literature and Linguistic Fiction. "International Journal of the Sociology of Language, 15, pp. 91-102.

BAKHTINE, Mikhail et V.N. Volochinov (1977). Le Marxisme et la philosophie du langage : essai d'application de la méthode sociologique en linguistique, trad. Marina Yaguello. Paris, Minuit, coll. "Le sens commun ".

BALZAC, Honoré de (1927). Euvres complètes - La Comédie humaine, éd. Marcel Bouteron, t. 31 (Études philosophiques, 5). Paris, Conard.

(1963). Le père Goriot, éd. Pierre-Georges Castex. Paris, Garnier, coll. « Classiques ».

BARTHES, Roland (1970). S/Z. Paris, Seuil, coll. « Points ".

(1982). "L'effet de réel " [1968], AAVV. Littérature et réalité. Paris, Seuil, coll. « Points 》, pp. 81-90. 
BASTIAENSEN, Michel (1974). "La rencontre de Panurge ", Revue belge de philologie et d'histoire, 52(3), pp. 544-565.

CHAITIN, Gilbert D. (1990). " Victor Hugo and the Hieroglyphic Novel », Nineteenth-Century French Studies, 19(1), pp. 36-53.

CHAMPOLLION, Jean-François (1994). " Lettre à M. [Joseph] Dacier relative à l'alphabet des hiéroglyphes phonétiques " [1822], in Gabriel Bergounioux, comp. Aux origines de la linguistique française. Paris, Pocket, coll. " Agora/Les classiques », pp. 92-98.

DUBOIS, Claude-Gilbert (1992). « Panurge-Pangloss. L'enfant trouvé à la langue perdue ", Europe, 70(757), pp. 58-66.

ELWERT, Wilhelm Theodor (1960). " L'emploi des langues étrangères comme procédé stylistique ", Revue de littérature comparée, 34(3), pp. 409-437.

FLAUBERT, Gustave (1979). Borvard et Pécuchet, avec un choix de scénarios, du Sottisier, de l'Album de la Marquise et du Dictionnaire des idées reçues, éd. Claudine Gothot-Mersch. Paris, Gallimard, coll. « Folio ».

FOUCAULT, Michel (1966). Les mots et les choses : une archéologie des sciences humaines. Paris, Gallimard, coll. « Tel ».

GAUVIN, Lise et Rainier Grutman (1996). « Langues et littératures: éléments de bibliographie », Littérature, 101, pp. 88-125.

GIESE, Wilhelm (1961). « El empleo de lenguas extranjeras en la obra literaria ", Studia Philologica : Homenaje ofrecido a Dámaso Alonso [...]. Madrid, Gredos, v. 2, pp. 79-90.

GOETSCH, Paul, dir. (1987). « Fremdsprachen in der Literatur. Ein typologischer Überblick ", in Paul Goetsch, dir. Dialekte und Fremdsprachen in der Literatur. Tubingen, Narr, coll. "ScriptOralia ", pp. 43-68. 
GRUTMAN, Rainier (1990). " Le Bilinguisme littéraire comme relation intersystémique ", Revue canadienne de littérature comparée, 17(3-4), pp. 198-212.

(1993). " Mono vs Stereo : Bilingualism's Double Face ", Visible Language, 27(1-2), pp. 206-227.

(1994). «Métalangage et drame hugolien », Texte, 15-16, pp. 211-227.

GUSDORF, Georges (1993). Le Romantisme, vol. I : Le Savoir romantique. Paris, Payot et Rivages.

HORN, András (1981). " Ästhetische Funktionen der Sprachmischung in der Literatur ", Arcadia, 16, pp. 225-241.

HUGO, Victor (1985). CEuvres complètes : Roman I, éd. Jacques Seebacher. Paris, Laffont, coll. « Bouquins ».

IRWIN, John T. (1980). American Hieroglyphics: The Symbol of Egyptian Hieroglyphics in the American Renaissance. New Haven, Yale University Press.

JEANNERET, Michel (1984). " Polyphonie de Rabelais : ambivalence, antithèse et ambigulité ", Littérature, 55, pp. 98-111.

KLEIN-LATAUD, Christine (1991). Précis des figures de style. Toronto, Éditions du GREF, coll. «Traduire, Écrire, Lire ».

KREMNITZ, Georg (1987). « Diglossie : possibilités et limites d'un terme ", Lengas, $11(22)$, pp. 199-213.

LAWRENCE, D.H. (1960). Women in Love. Harmondsworth, Penguin.

MARCH, Kathleen N. (1984). "El bilingüismo literario y la verosimilitud ", Revista de literatura, 46(92), pp. 109-116. 
MAUPASSANT, Guy de (1971). "Étude sur le roman. Préface à Pierre et Jean [1887] ». in Herbert S. Gershman et Kerman B. Whitworth, comp. Anthologie des préfaces de romans français du $X I X^{*}$ siècle. Paris, Éditions UGE, coll. « 10/18 », pp. 362-380.

PRUDENT, Lambert-Félix (1981). " Diglossie et interlecte ", Langages, 61, pp. 13-38.

RABELAIS, François (1994). Les cinq livres : Gargantua, Pantagruel, Le tiers livre, Le quart livre, Le cinquième livre, éd. Jean Céard, Gérard Defaux et Michel Simonin. Paris, Le livre de poche, coll. «Classiques Modernes ».

RIGOLOT, François (1976). " Cratylisme et Pantagruélisme : Rabelais et le statut du signe ", Études rabelaisiennes, 13, pp. 115132.

SARKONAK, Ralph et Richard G. Hodgson (1993). " Seeing in Depth : the Practise of Bilingual Writing ", Visible Language, 27(12), pp. 6-39.

STERNBERG, Meir (1981). " Polylingualism as Reality and Translation as Mimesis ", Poetics Today, 2(4), pp. 221-239.

ULLMANN, Stephen (1964). Style in the French Novel. Oxford, Blackwell.

RÉSUMÉ: Langues étrangères et savoir romantique : considérations préliminaires - La valeur et la signification de la cohabitation textuelle des langues changent trop au gré des attitudes épilinguistiques pour qu'il soit possible, ou même salutaire, de les subsumer sous le dénominateur commun du " réalisme ". La lecture référentielle, en effet, ne rend guère compte des manières dont les langues se font écho, se complètent, se traduisent à l'intérieur de l'œuvre littéraire. Or, loin de simplement enregistrer le social, celleci formule un savoir sur les idiomes mis à contribution, ce qu'illustre une brève comparaison des conceptions « classique " et 
" romantique " du plurilinguisme (à partir du Pantagruel de François Rabelais et Notre-Dame de Paris de Victor Hugo). Alors que dans le premier cas, le xénisme fait obstacle en court-circuitant le rapport dénotatif entre les mots et les choses, dans le deuxième, il s'insère dans un réseau de connotations propres au texte.

\begin{abstract}
Foreign Languages and Romantic Knowledge : Some Preliminary Considerations - Because its meaning varies so greatly in accordance with prevailing language attitudes, textual multilingualism is too many-sided a phenomenon to be easily subsumed under the heading of " realism "). Referent-oriented readings cannot explain how languages interact with, complete, and translate each other within the boundaries of texts which make an informed use of foreign tongues instead of simply mirroring society. The latter point is illustrated with a brief comparison between " classical " and " romantic " conceptions of multilingualism in French literature. Whereas in François Rabelais' Pantagruel, foreignisms short-circuit the referential link between words and things, they can be said to build an internal network of connotations in Victor Hugo's Notre-Dame de Paris.
\end{abstract}

Rainier Grutman: Département des lettres françaises, Université d'Ottawa, C.P. 450, Succursale A, Ottawa (Ontario) K1N 6N5 CANADA 